\title{
POLIETILENO TIBIAL MÓVEL NA ARTROPLASTIA TOTAL DO JOELHO
}

\section{MOBILE POLYETHYLENE BEARING IN TOTAL KNEE REPLACEMENT}

\author{
Hugo Alexandre de Araújo Barros Cobra', Idemar Monteiro da Palma²
}

\section{RESUMO}

O desgaste do polietileno tibial utilizado nas artroplastias de joelho origina partículas que, quando fagocitadas, dão início à cascata de eventos biológicos que levam à osteólise e consequente afrouxamento dos componentes da prótese. Assim sendo, alternativas para o polietileno têm sido pesquisadas com o objetivo de minimizar o desgaste e, com isso, aumentar a durabilidade das artroplastias. Uma dessas opções é a utilização de polietilenos tibiais móveis, que apresentam maior conformidade do que os polietilenos fixos, ao mesmo tempo em que permitem autoalinhamento rotacional entre os componentes, melhorando, dessa forma, a cinética e a cinemática da prótese. São apresentados aqui, de forma resumida, porém abrangente, o conceito, os fundamentos biomecânicos, as indicações, os resultados esperados e complicações dos polietilenos tibiais móveis nas artroplastias totais do joelho.

Descritores - Joelho; Biomecânica; Artroplastia; Polietileno

\section{ABSTRACT}

Debris of polyethylene tibial bearings have been recognized as a major cause for the onset of the cascade of biological events leading to osteolysis and loosening of prosthetic components after total knee arthroplasty. Since then, research has been focused on alternative bearing surfaces in order to minimize the amount and rate of polyethylene wear off and, in doing so, increasing the survivorship rate for knee arthroplasties. One such option is to have a mobile tibial bearing allowing more conformity and rotational self-alignment of the components, improving kinetics and kinematics of the prosthesis. The authors present a resumed but throughout and comprehensive review of the rationale, biomechanics fundamentals, indications, pitfalls, outcomes and complications for the use of mobile tibial bearings in total knee replacement.

Keywords - Knee; Biomechanics; Arthroplasty; Polyethylene

\section{INTRODUÇÃO}

Reconhecido como uma das principais causas de falha nas artroplastias totais do joelho, o polietileno tibial tem sido o foco de diversas pesquisas, desde novos métodos de manufatura a formas de sua utilização, objetivando diminuir o nível de desgaste do material e suas consequências ${ }^{(1,2)}$.

Uma das alternativas adotadas é a utilização de polietilenos tibiais móveis, mais congruentes, que diminuiriam não só o desgaste da superfície superior, em contato direto com o componente femoral, como também o desgaste observado na superfície inferior dos polietilenos fixos ${ }^{(2-4)}$.

Outra vantagem seria a possibilidade de o polietileno móvel se autoposicionar, permitindo e acomodando erros no posicionamento rotacional do componente tibial ${ }^{(2,4-7)}$.

Atualmente, todas as grandes companhias que produzem e comercializam os componentes das artroplastias totais de joelho têm em seu portfólio a alternativa de utilização do polietileno tibial móvel.

Entretanto, também há desvantagens a considerar. $\mathrm{O}$ custo de uma artroplastia total de joelho com plataforma móvel é maior do que a com componente de polietileno fixo. A possibilidade de luxação rotatória do polietileno móvel (spin-out) tem que ser avaliada e prevenida, pois pode levar à necessidade de cirurgia de revisão ${ }^{(4,8)}$.

\section{ASPECTOS BIOMECÂNICOS}

Componentes de polietileno tibial móvel não são novidade, sendo o primeiro a ser utilizado o da artroplastia unicompartimental tipo Oxford (Biomet, Bridgend, South Wales) há 30 anos, logo acompanhado pelo lançamento do sistema LCS (Low Contact Stress) (DePuy, Warsaw, Indiana) ${ }^{(2,9)}$.

O conceito de polietileno tibial móvel é atrativo e pode ser a solução de alguns problemas e dilemas biomecânicos das artroplastias do joelho.

Como ocorre rotação entre a bandeja tibial e o polietileno, a necessidade de permitir rotação ao nível da arti-

1 - Médico Ortopedista do Grupo de Joelho do Instituto Nacional de Traumatologia e Ortopedia do Ministério da Saúde, Rio de Janeiro - RJ.

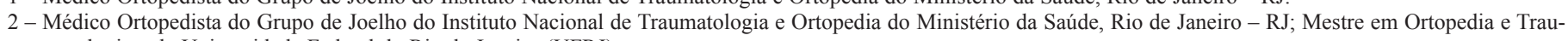
matologia pela Universidade Federal do Rio de Janeiro (UFRJ).

Correspondência: Praia do Flamengo, 66, bloco B, sala 1.313 -22210-030 - Rio de Janeiro, RJ. E-mails: hmcobra@uol.com.br e idemont@terra.com.br 
culação femorotibial, como tem que ocorrer nas plataformas fixas, é eliminada e a área de contato é aumentada de $200 \mathrm{~mm}^{2}$ para $1.000 \mathrm{~mm}^{2}$ ou mais, com consequente diminuição das forças de estresse de contato ${ }^{(2,5,6,9,10)}$.

Por outro lado, diferentemente dos polietilenos fixos iniciais que, para permitir rotação entre o fêmur e a tíbia, apresentavam superfície de contato femoral plana, reta, levando a maiores forças de estresse de contato, especialmente do tipo pontual, a maior conformidade do polietileno móvel aumenta e mantém a área de contato durante todo o arco de movimento.

Sendo assim, o conflito cinemático (superfície/rotação) observado com as plataformas fixas pode ser resolvido, já que uma superfície articular de alta conformidade passa a poder coexistir com movimento rotacional ${ }^{(2,6,7,11)}$.

Isso levaria, em última análise, a menos atrito e menor desgaste do polietileno, duas variáveis tribológicas importantes, com consequente aumento de durabilidade da prótese.

As plataformas de polietileno tibiais móveis, quando pareadas aos componentes metálicos, isto é, quando os componentes femoral, tibial e o polietileno são do mesmo tamanho, permitem rotação interna ou externa de aproximadamente $10^{\circ}$, sem haver protrusão do polietileno sobre a bandeja metálica tibial. Em casos onde se faz necessária a utilização de um componente de polietileno tibial maior do que a bandeja tibial metálica, ainda assim é possível observar rotação interna e externa do polietileno em torno de $5^{\circ}$, também sem haver protrusão do polietileno ${ }^{(6)}$.

As plataformas móveis podem ser descritas, em relação à sua mobilidade, como sendo de rotação pura (por exemplo, a plataforma rotatória LCS (Figura 1), de translação anteroposterior (por exemplo, a prótese unicompartimental tipo Oxford) (Figura 2), rotação com translação anteroposterior (por exemplo, os meniscos de polietileno móveis da LCS (Figura 3).

É importante ressaltar que, para haver mobilidade entre o polietileno e a bandeja tibial, é necessário que a superfície metálica superior do componente tibial seja completamente uniforme e lisa, livre de rugosidades, evitando-se assim o atrito e consequente desgaste. Por essa razão, os componentes tibiais são de cromo-cobalto, já que com as ligas de titânio não se consegue, até o momento, a eliminação das rugosidades de superfície.

\section{INDICAÇÕES E TÉCNICA CIRÚRGICA}

As indicações para a utilização dos componentes de polietileno tibial móvel são, na verdade, as mesmas para artroplastia total de joelho com componentes de polietileno tibial fixo. Entretanto, devido a seu custo mais elevado e pela possibilidade de maior durabilidade, a tendência é a de reservar seu uso para os pacientes mais jovens e mais ativos ${ }^{(6)}$. Na verdade, essa escolha acaba ficando na dependência da preferência e experiência do cirurgião e, também, obviamente, da disponibilidade da prótese.

A técnica cirúrgica é semelhante à empregada para a realização de uma artroplastia total de joelho com plataforma fixa. Há que se ter especial atenção nos espaços de flexão e extensão, pois, se desiguais, podem ser uma das causas de luxação rotatória do polietileno móvel. Outro fator importante na prevenção dessa luxação rotatória do polietileno móvel é a tensão do ligamento cruzado posterior, que deve ser verificada através do teste do deslizamento posterior, conforme descrito por $\operatorname{Scott}^{(6)}$. Essa verificação é feita com os componentes de teste metálicos femoral e tibial e o de polietileno tibial devidamente posicionados. Deve-se ter o cuidado de o polietileno tibial estar apoiado livre sobre a bandeja metálica tibial de teste. O joelho é, então, colocado entre $80^{\circ}$ e $100^{\circ}$ de flexão, verificando-se o deslocamento do componente de polietileno tibial.

Se houver deslocamento do polietileno tibial para frente da bandeja metálica tibial, além de tendência a, ou mesmo, levantamento dessa bandeja metálica tibial, há tensão exagerada do ligamento cruzado posterior.
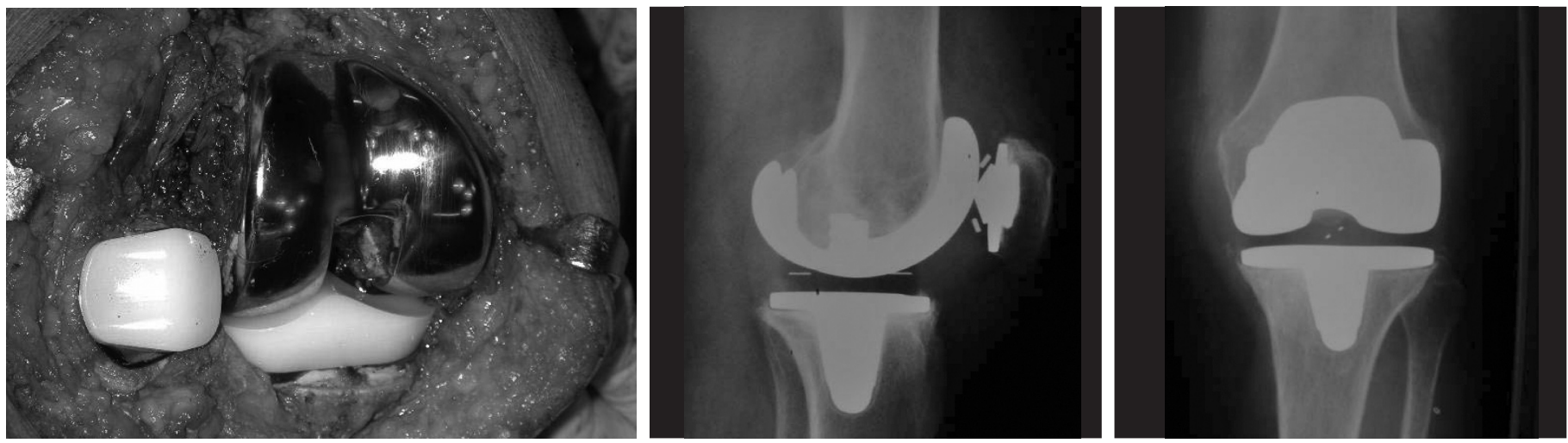

Figura 1 - Aspecto cirúrgico de plataforma de polietileno tibial móvel. Notar, também, o componente patelar móvel. Abaixo, imagens radiológicas em perfil e AP. Notar os marcadores radio-opacos, tanto no polietileno tibial quanto no patelar. 


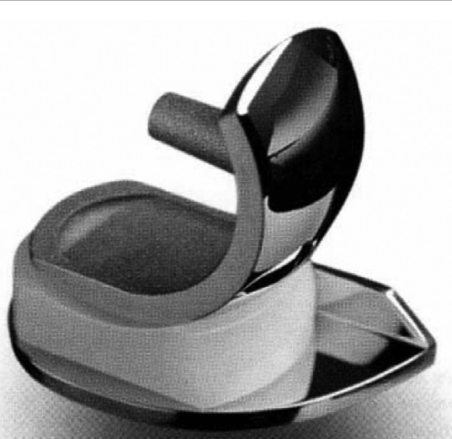

Figura 2 - Imagem da prótese unicompartimental tipo Oxford, série 3.

Caso o polietileno tibial se desloque posteriormente mais do que $3 \mathrm{~mm}$, isso é sinal de frouxidão do ligamento cruzado posterior.

O ideal é que, nessa situação, o polietileno tibial esteja situado numa posição 2 a $3 \mathrm{~mm}$ posterior à borda anterior da bandeja metálica tibial, denotando tensão adequada do ligamento cruzado posterior.

\section{RESULTADOS}

Os resultados das artroplastias totais do joelho com plataformas móveis, obrigatoriamente, têm que ser com- parados com os obtidos com a utilização de plataformas fixas $^{(2,6,9,12,13)}$. As artroplastias totais de joelho com plataforma fixa oferecem resultados subjetivos e objetivos expressivos e com índice de durabilidade de mais de $90 \% \mathrm{em}$ longo prazo (15 anos ou mais) $)^{(1,2,6)}$. As artroplastias totais com plataformas móveis tipo LCS também demonstraram resultados comparáveis no seguimento em longo $\operatorname{prazo}^{(9)}$.

Além das complicações gerais inerentes aos procedimentos artroplásticos, a utilização do polietileno tibial móvel apresenta algumas complicações específicas.

Pode haver maior possibilidade de desenvolvimento de instabilidade posterolateral devido à maior translação do polietileno móvel no sentido anteroposterior do que o que se observa nas plataformas fixas. Pode, também, haver impacto do compartimento anterior do joelho, especialmente se o panículo adiposo patelar não for ressecado apropriadamente.

A complicação mais comum é a luxação rotatória do polietileno (spin-out), que pode ser parcial ou total (Figuras 4, 5, 6, 7 e 8). E essa é uma complicação diretamente relacionada a erro técnico, em outras palavras, ao cirurgião.

Com o objetivo de não permitir a ocorrência dessa luxação rotatória, alguns desenhos incorporam pinos metá-
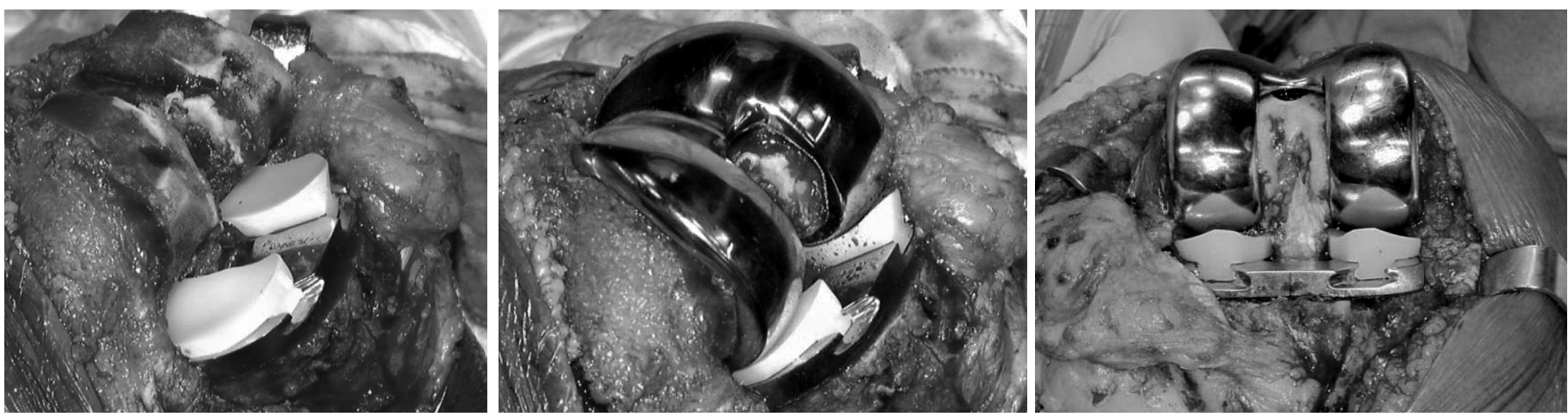

Figura 3 - Bandeja metálica tibial com preservação do LCP, para utilização de meniscos de polietileno móveis. Observar os trilhos na bandeja metálica para os meniscos de polietileno.

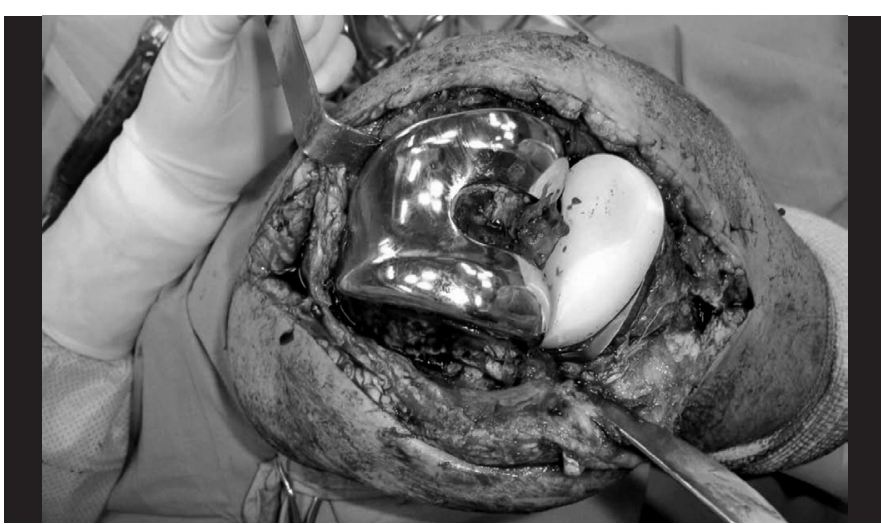

Figura 4 - Detalhe cirúrgico demonstrando início do fenômeno de luxação rotatória ("spin-out") do polietileno tibial móvel.

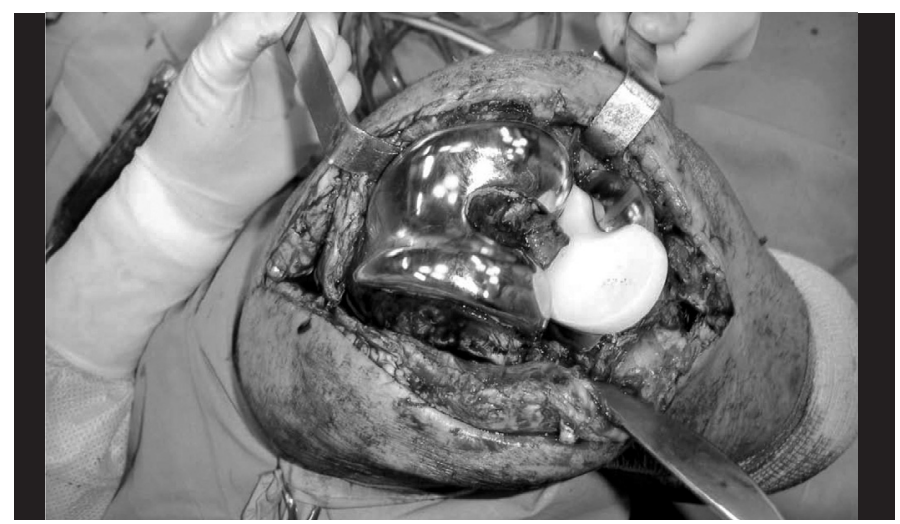

Figura 5 - Sequência da luxação rotatória do polietileno tibial móvel. Notar sua posição transversal em relação aos componentes metálicos. 


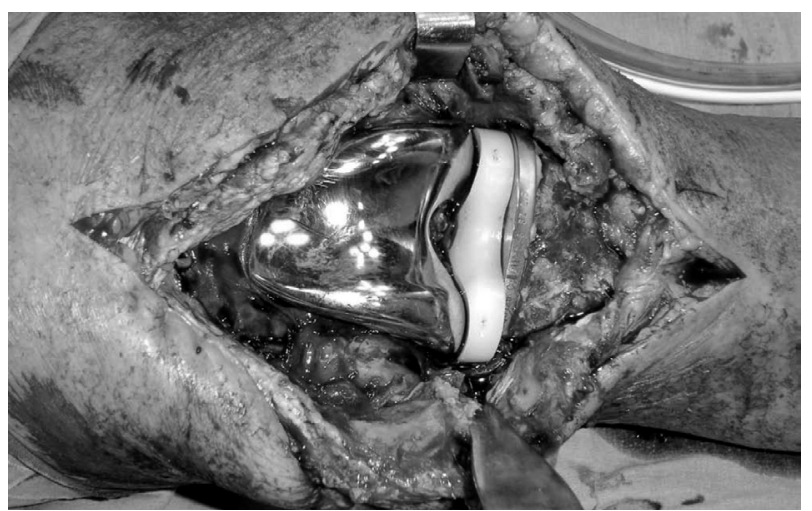

Figura 6 - Luxação rotatória completa. Detalhe com o joelho em extensão. Notar a inversão completa do polietileno tibial.

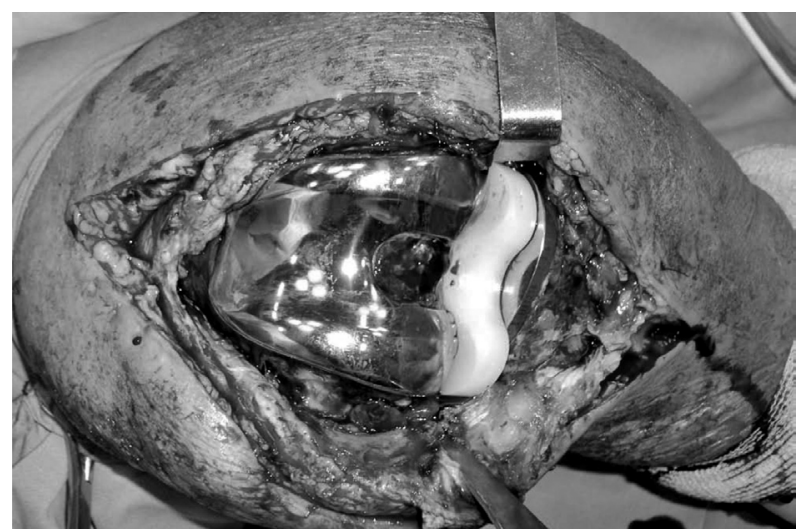

Figura 7 - Luxação rotatória completa. Detalhe com o joelho em flexão. Notar a discrepância rotacional entre a bandeja metálica tibial e o polietileno.

licos à bandeja tibial, um anterior e outro posterior. Entretanto, isso pode levar à diminuição do grau de rotação do polietileno e,à possibilidade de maior atrito e desgaste.

\section{CONCLUSÃO}

A utilização de plataformas tibiais móveis tem grande apelo do ponto de vista biomecânico, especialmente em relação ao menor desgaste do polietileno, e também

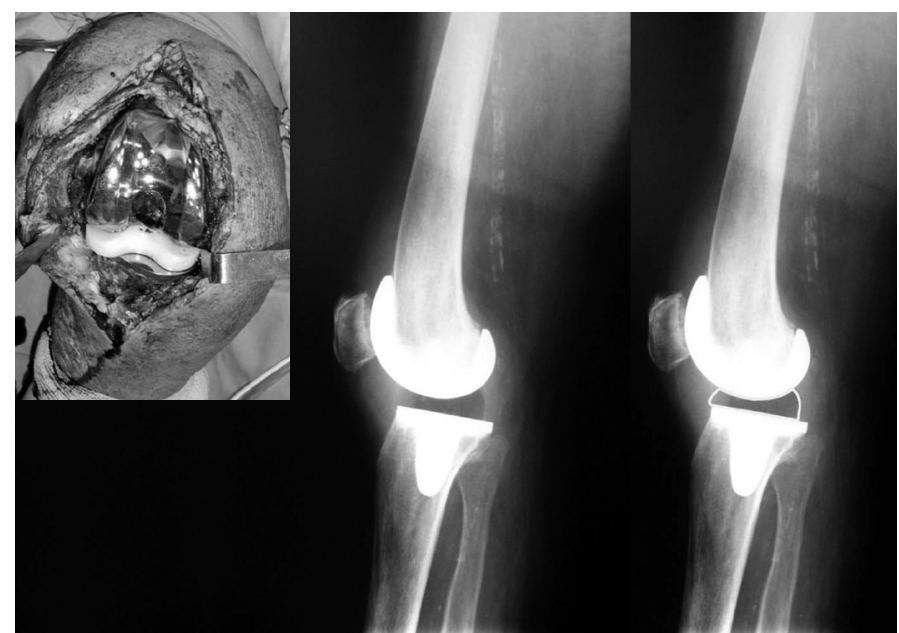

Figura 8 - Aspecto radiológico em perfil da luxação rotatória ("spin-out") completa do polietileno tibial. Nota-se a inversão completa do polietileno, especialmente no delineamento feito à direita. Imagem cirúrgica inserida para comparação.

por permitir autoajuste rotacional entre o polietileno e a bandeja tibiais. Entretanto, não podemos esquecer a possibilidade de luxação rotatória do polietileno, que pode levar à necessidade de revisão.

Os princípios cirúrgicos básicos da artroplastia total do joelho, especialmente os relacionados com a simetria dos espaços de flexão e extensão e com o correto reequilíbrio ligamentar, tem que ser respeitados. Caso contrário, independente do tipo de prótese ou polietileno utilizados, comprometemos, certamente, o resultado da cirurgia.

Fator muito importante em nosso meio é o custo elevado em relação às próteses com plataforma fixa, além da dificuldade de disponibilidade do sistema, o que acaba inviabilizando sua utilização mais larga.

Esperamos que, em futuro próximo, esses obstáculos sejam superados para que possamos contar com mais essa alternativa cirúrgica para o tratamento da doença articular degenerativa dos joelhos.

\section{REFERÊNCIAS}

1. Horowitz SM. Particulate debris and osteolysis. In: Lotke PA, Garino JP, editors. Revision total knee arthroplasty. Philadelphia: Lippincot-Raven Publishers; 1999.p.31-41.

2. Callahan JJ, Insall JN, Greenwald AS, Douglas DA, Komistek RD, Murray DW, et al. Mobile bearing knee replacement: concepts and results. J Bone Joint Surg Am. 2000;82(7):1020-41

3. Wasielewski RC, Parks N, Williams I, Surprenant H, Collier J, Engh G. Tibial insert undersurface as a contributing source of polyethylene wear debris. Clin Orthop Relat Res. 1997;(345):53-9.

4. Otto JK, Callaghan JJ, Brown TD. Mobility and contact mechanics of a rotating platform total knee replacement. Clin Orthop Relat Res. 2001;(92):24-37.

5. Dennis DA, Komistek RD, Mahfouz MR, Outten JT, Sharma A. Mobile-bearing total knee arthroplasty. Do the polyethylene bearings rotate? Clin Orthop Relat Res. 2005;(440):88-95.

6. Scott RD. Mobile versus fixed bearings in total knee arthroplasty. In: Total knee arthroplasty. Philadelphia: Saunders, 2006;16-9.

7. Most E, Li G, Schule S, Sultan P, Park SE, Zayontz S, Rubash HE. The kine-

matics of fixed- and mobile-bearing total knee arhroplasty. Clin Orthop Relat Res. 2003;(416):197-207.

8. Huang $\mathrm{CH}$, Ma HM, Liau JJ, Ho FY, Cheng CK. Late dislocation of rotating platform in New Jersey Low Contact Stress knee prosthesis. Clin Orthop Relat Res. 2002;(405):189-94.

9. Buechel FF Sr, Buechel FF Jr, Pappas MJ, D`Alessio J. Twenty-year evaluation of meniscal bearing and rotating platform knee replacements. Clin Orthop Relat Res.2001;(388):41-50.

10. Dennis DA, Komistek RD, Mahfouz MR. In vivo fluoroscopic analysis of fixedbearing total knee replacements. Clin Orthop Relat Res. 2003;(410):114-30.

11. Banks S, Bellemans J, Nozaki H, Whiteside LA, Harman M, Hodge, WA. Knee motions during maximum flexion in fixed and mobile-bearing arthroplasties. Clin Orthop Relat Res. 2003;(410):131-8.

12. Chiu KY, Ng TP, Tang WM, Lam P. Bilateral total knee arthroplasty: one mobilebearing and one fixed-bearing. J Orthop Surg. 2001;9(1):45-50.

13. Kim JS. Comparison of fixed-bearing and mobile-bearing total knee arthroplasties. Clin Orthop Relat Res. 2001;(392):101-15. 